\title{
Effects of Dual-Task Conditions on Turning Characteristics of Stroke Survivors in Healthcare Environment
}

\author{
Haidzir Manaf ${ }^{1 *}$, Nadia Mohd Mustafah ${ }^{2}$, Saiful Adli Bukry ${ }^{1}$, Maria Justine ${ }^{1}$ \\ ${ }^{1}$ Department of Physiotherapy, Universiti Teknologi MARA, Puncak Alam Campus, 42300, Puncak Alam, Malaysia \\ ${ }^{2}$ Department of Rehabilitation Medicine, Universiti Teknologi MARA, 47000, Sg Buloh, Malaysia
}

\begin{abstract}
Turning is an attentionally demanding task for stroke survivors especially when it involves challenging environments. The aim of this study was to investigate turning characteristics under dual-task conditions (dual motor and dual cognitive) in stroke survivors and healthy controls. We compared the turning characteristics based on the following: (1) staggering, (2) number of steps, (3) time was taken, and (4) strategy used to complete a $180^{\circ}$ turn. We found that stroke survivors presented with turning difficulty, especially under dual cognitive task. Forthcoming research is needed to investigate further the social impact of stroke survival in their current environment.

(C) 2016. The Authors. Published for AMER ABRA by e-International Publishing House, Ltd., UK. Peer-review under responsibility of AMER (Association of Malaysian Environment-Behaviour Researchers), ABRA (Association of Behavioural Researchers on Asians) and cE-Bs (Centre for Environment-Behaviour Studies, Faculty of Architecture, Planning \& Surveying, Universiti Teknologi MARA, Malaysia. .
\end{abstract}

Keywords: Dual-task; stroke; turning; Timed Up \& Go test

\section{Introduction}

A walkable environment allows people to experience walking on foot safely. The environmental qualities that enable walking determine an individual's sense of comfort and safety (Zakaria \& Ujang, 2015). In Malaysia, the implementations of the barrierfree facilities are not well established (Osman, Radzi, Bakri, \& Ibrahim, 2015). This may lead to fall incidence among persons with a disability such as stroke survivors. Walking in the outdoor environment should be legible, distinctive, accessible, comfortable and safe for individuals with a disability (Abbas \& Saruwono, 2012; Mohammad \& Abbas, 2012). Therefore, it is important to understand the factors that can cause falls among stroke survivors, especially when it involves challenging environments as well as multiple activities.

Falls is one of the most common complications among stroke survivors. Approximately $50 \%$ of stroke survivors experience at least one fall incidence in a year. Concerns have been raised about the safety while walking especially in the community or outdoor environment (Eusuf, Mohit, Eusuf, \& Ibrahim, 2014). Most of the falls happened during walking, especially during outdoor walking, obstacle avoidance and turning (Yates, Lai, Duncan, \& Studenski, 2002). In fact, turning activity involves $35 \%$ - $45 \%$ of

\footnotetext{
${ }^{*}$ Corresponding author. Tel.: +603-3258-4376; fax: +03-3258-4599.

E-mail address: haidzir5894@puncakalam.uitm.edu.my.
} 
the steps taken during walking. In stroke survivors, turning related falls often occur when turning towards the paretic side. Falls may result in more serious consequences and deterioration in physical health (Cumming \& Klineberg, 1994; Hyndman, Ashburn, \& Stack, 2002). Therefore, it is critical to examine the turning difficulty characteristics in stroke survivors such that appropriate prevention can be taken.

A previous study has used Timed Up and Go (TUG) test to capture turning difficulty characteristics in elderly (Thigpen, Light, Creel, \& Flynn, 2000). They identified four indicators of turning difficulty characteristics: (1) staggering, (2) absence of pivoting, (3) taking five or more steps, and (4) taking $3 \mathrm{sec}$ or longer to achieve the turn. Another study has found that the time and number of steps to complete $180^{\circ}$ turn were moderately correlated with postural control performance (Chen, Chen, Huang, Chung, \& Tang, 2007). This indicates that individuals with poor postural control need more steps and longer time to complete turning. Also, TUG test consist of dual-task components that are the ability to perform two different tasks at the same time (Shumway-Cook, Brauer, \& Woollacott, 2000). Therefore, the dual task conditions further challenge gait stability and provide a more comprehensive assessment of balance capacity during turning.

Turning is not an automatic process. Skillful turning requires higher attention function, modifications of gait parameters, and reorientation of the body to a new direction. Therefore, attention control is necessary to ensure that all of these steps are monitored and operated for safely executing a turn while walking. Attention is conceptualized as the information-processing capacity needed to perform a task, and it can be categorized into sustained, switching, selective and divided attention (YogevSeligmann, Hausdorff, \& Giladi, 2008). In this study, we primarily focused on divided attention, which refers the ability to carry out more than one task at the same time. Dual-task interference occurs when the task performance requires an attentional resource that exceeds the capacity. For example, performing both the walking task and secondary task may congest the attention capacity. If a person is given the instruction to prioritize the performance of the secondary task (counting backward), in which walking will be compromised to optimize performance on the secondary task. As a result, stroke survivors may demonstrate deterioration in gait performance when they perform two tasks simultaneously.

Stroke survivors with impaired sensorimotor and balance performance may have difficulty to perform safe turning, especially under dual-task conditions. To date, there is still a lack of research data on turning difficulty characteristics under dual-task conditions. The need for research into the safe walking environment is particularly essential in community-dwelling chronic stroke survivors that might influence their quality of life (Mohit, 2014). The aim of this study is to have a better understanding of the turning deficits among stroke survivors. Exploration of turning difficulty characteristics under dual-task conditions is important for guiding the development of an intervention for stroke survivors who have a high risk of falls. While at the same time trying to encourage functional ambulation especially in the barrier-free community environment (Osman, Radzi, Bakri \& Ibrahim, 2015; Samah, Ibrahim, \& Amir, 2013). Therefore, the objective of this study was to examine the turning difficulty characteristics in stroke survivors and healthy controls dual-task conditions. We hypothesized that stroke survivors presented with turning difficulty under dual-task conditions.

\section{Methods}

Thirteen stroke survivors and 12 healthy controls participated in this study. The calculation of sample size in this study was done using the G-Power 3 software. Power analysis using our previous study that investigated the TUG performance among individuals with stroke showed that an estimated sample of 20 participants would provide $80 \%$ power with a risk of type 1 error of 0.05 . Stroke survivors were recruited from a government-funded hospital using a convenience sampling. The inclusion and exclusion criteria were examined based on the medical record and clinical assessment done by the rehabilitation medicine physician. The inclusion criteria for stroke survivors were as follows: (1) > 6-month post stroke, (2) able to walk independently while holding a glass of water, (3) able to understand and follow instructions, and (4) able to do simple arithmetic calculation such as count backward from 20 by three $(17,14,11,8,5,2)$. Both stroke survivors and healthy controls were excluded if they were presented with other neurologic disorders (other than stroke), severe musculoskeletal condition and cognitive dysfunction (scored 24 or less at the Mini-Mental State Examination). All participants signed an informed consent form approved by the institutional ethics committee prior to participation.

Prior to the testing, participants were briefed about the purpose, design, method of the study, confidentiality, right to withdraw without penalty, and benefits and risks of participation before signing the informed consent. Then, the participants' demographic data were recorded. In addition, we measured their cognitive function with the Mini-Mental State Examination, stroke severity with the Fugl-Myer Assessment (FMA) and balanced with the Berg Balance Scale (BBS). Data collection was conducted in a 
physiotherapy gymnasium. Safety mat was placed in front of the turning area for safety precautions during $180^{\circ}$ turning. Other than that, tester stood by the participant's side to prevent falls during the procedure

In this study, participants were asked to perform Timed Up and Go test (TUG) under three conditions: (1) single task (without any secondary task), (2) dual motor task (holding a glass full of water with the non-affected side), and (3) dual cognitive task (a counting backward task). The TUG test is a performance-based tool used to assess the balance and mobility performance in populations with various diagnoses. The TUG test measures the time taken for an individual to stand up from a chair, walk 3 meters, turn $180^{\circ}$, walk 3 meters back to the chair, and sit down. Turning $180^{\circ}$ was selected in this study that able identify gait impairment in turning ability with the larger end of degrees. These degrees have been identified of having more than threequarters of all turns in frequent daily tasks.

For the dual motor task condition, participants were instructed to perform TUG test while holding the glass without spilling the water (prioritized glass holding). If participants spilled the water, the trial would be considered as a failed trial and be repeated. For dual cognitive task conditions, participants were verbally given a number (any number from 50 to 100). Then they performed TUG test while counted backward by three from the number consecutively and gave verbal responses. For example, if the given number was 80 , participants responded as "77, 74, 71..." Trials were repeated three times. During the test, participants had to count continuously; if they did not, it would be considered as a failed trial and be repeated. The testing procedure was recorded using a digital video camera (30 frames per second) that mounted on the tripod in the sagittal plane. Participants were instructed to perform three trials for each condition and prioritized the secondary task (motor or cognitive task). The testing trials were randomized among participants. A 5-min rest was given between testing conditions to avoid fatigue.

We assigned four assessors with working experience as a physiotherapist in a stroke rehabilitation (> five years) to view and rate the videos. They were given a form that has information regarding the turning qualities of participant, which include: 1) staggering, 2) number of steps, 3) time was taken, and 4) type of strategy used to complete turn (Thigpen et al., 2000). The recorded video viewed in the sagittal plane of movement using a digital video player with frame-by-frame slow-motion capabilities. The beginning of turn was defined as the time when the foot first contacted the turning area after walking for 3-meter and ended when the foot left the turning area. During the video analysis, no discussion occurred between the physiotherapists.

From the recorded video, the following outcomes (dependent variables) were obtained: time and number of steps taken to complete the TUG test. The independent variables were attentional loading condition (single and dual motor task conditions) and turning direction (non-paretic and paretic sides). We averaged three trials for each condition and further divided the TUG test into turning and straight walking phases to determine whether attentional loading had a differential effect on each phase. The data were analyzed using the SPSS statistical software (version 19.0, SPSS Inc., USA). Before data analysis, raw data were screened for error and validated by the researcher. Once there were no errors in the data, researcher started to analyze the data. Data were checked for normal distribution with a statistical normality test (Shapiro-Wilk), skewness and kurtosis. Mean, standard deviation and range were calculated for each variable. The independent samples t-test was used to compare the demographic data between stroke survivors and healthy controls. The percentage of turning qualities was calculated to represent the frequency difficulty in turning while turning in stroke survivors and healthy controls.

\section{Results}

Demographic data are presented in Table 1. The data were collected from 13 stroke survivors (10 males, 3 females) and 12 healthy controls ( 10 males and 2 females). Among the 13 stroke survivors, 5 participants were presented with right hemiparesis with the mean duration of post-stroke was 17.3 months.

Table 1. Demographic characteristics of the study participants

\begin{tabular}{lll}
\hline Characteristics & Stroke Survivors $(\mathrm{n}=13)$ & Healthy Control $(\mathrm{n}=12)$ \\
\hline Age $($ years $)$ & $49.3 \pm$ & $52.8 \pm$ \\
Body weight $(\mathrm{kg})$ & $65.9 \pm$ & $74.1 \pm$ \\
Body height $(\mathrm{meter})$ & $1.7 \pm$ & $1.7 \pm$ \\
Body Mass Index $(\mathrm{BMI})\left(\mathrm{kg} / \mathrm{m}^{2}\right)$ & $24.0 \pm($ & $26.0 \pm$ \\
Mini Mental State Examination & $28.7 \pm$ & $29.5 \pm$ \\
\hline
\end{tabular}


(Max score 30)

\subsection{Staggering during turning}

Table 2 shows the percentage of staggering. The majority of the stroke survivors were presented with no staggering during $180^{\circ}$ turning that is $92.3 \%$ in single and dual motor task while $84.6 \%$ in the dual cognitive task. Only 1 to 2 participants with $7.7 \%$ in single and dual motor task as well as $15.4 \%$ in the dual cognitive task are having loss of balance during the turn but have selfcorrection without assistance. Despite that, all of the healthy controls obtain $100 \%$ with no staggering in all tasks.

Table 2. Percentage of staggering incidence during turn under dual-task conditions for stroke survivors and healthy controls

\begin{tabular}{|c|c|c|c|c|c|c|}
\hline Staggering & & Stroke & & & Healthy Control & \\
\hline Category & $\begin{array}{l}\text { Single-Task } \\
(\%)\end{array}$ & $\begin{array}{l}\text { Dual Motor Task } \\
(\%)\end{array}$ & $\begin{array}{l}\text { Dual Cognitive Task } \\
(\%)\end{array}$ & $\begin{array}{l}\text { Single-Task } \\
(\%)\end{array}$ & $\begin{array}{l}\text { Dual Motor Task } \\
(\%)\end{array}$ & $\begin{array}{l}\text { Dual } \\
\text { Cognitive } \\
\text { Task (\%) }\end{array}$ \\
\hline 0 -No loss of balance during turning & 92.3 & 92.3 & 84.6 & 100 & 100 & 100 \\
\hline $\begin{array}{l}\text { 1-Loss of balance during turning self- } \\
\text { correct without assistance }\end{array}$ & 7.7 & 7.7 & 15.4 & 0 & 0 & 0 \\
\hline $\begin{array}{l}\text { 2- Loss of balance during turning } \\
\text { requires guarding lassistance to } \\
\text { correct or prevent fall. }\end{array}$ & 0 & 0 & 0 & 0 & 0 & 0 \\
\hline
\end{tabular}

\subsection{Number of steps during $180^{\circ}$ turn}

Table 3 describes the number of steps taken during turning. Mostly, stroke survivors use 5 steps and more while turning with the score of $92.3 \%$ in a single task and $100 \%$ in both dual motor and dual cognitive task. Only $7.7 \%$ of them used 3 to 4 steps during turn in a single task. In contrast, the healthy controls were found to use 1 to 2 steps during turning that is $58.3 \%$ in single task, $50 \%$ in dual motor and more than 5 steps that are $41.7 \%$ in dual cognitive task. The other percent is $25 \%$ in the dual motor, and dual cognitive task meanwhile $16.7 \%$ in the single task is using 3 to 4 steps while turning. Lastly, $33.3 \%$ of healthy controls also used 1 to 2 steps during the dual cognitive task and $25 \%$ of single and dual motor task are using 5 steps and more.

Table 3. Percentage of number of steps to complete the turn under dual-task conditions for stroke survivors and healthy controls

\begin{tabular}{|c|c|c|c|c|c|c|}
\hline Number of Steps During Turning & \multicolumn{3}{|c|}{ Stroke } & \multicolumn{3}{|c|}{ Healthy Control } \\
\hline Category & $\begin{array}{l}\text { Single- } \\
\text { Task (\%) }\end{array}$ & $\begin{array}{l}\text { Dual Motor } \\
\text { Task }(\%)\end{array}$ & $\begin{array}{l}\text { Dual Cognitive } \\
\text { Task (\%) }\end{array}$ & $\begin{array}{l}\text { Single- } \\
\text { Task (\%) }\end{array}$ & $\begin{array}{l}\text { Dual Motor } \\
\text { Task (\%) }\end{array}$ & $\begin{array}{l}\text { Dual Cognitive } \\
\text { Task }(\%)\end{array}$ \\
\hline $\begin{array}{l}\text { 0-Accomplishes complete reversal of direction } \\
\text { with } 1 \text { to } 2 \text { steps involved in the turn }\end{array}$ & 0 & 0 & 0 & 58.3 & 50 & 33.3 \\
\hline $\begin{array}{l}\text { 1-Accomplishes complete reversal of direction } \\
\text { with } 3 \text { to } 4 \text { steps involved in the turn }\end{array}$ & 7.7 & 0 & 0 & 16.7 & 25 & 25 \\
\hline $\begin{array}{l}\text { 2- Accomplishes complete reversal of direction } \\
\text { with } 5 \text { steps or more involved in the turn }\end{array}$ & 92.3 & 100 & 100 & 25 & 25 & 41.7 \\
\hline
\end{tabular}




\subsection{Time taken during turning}

In most stroke survivors, they were found to use 1 second and above to complete turning in all tasks required. In contrast, the healthy controls obviously take 2.50 to 2.99 seconds to complete turning in single and dual motor task while $91.7 \%$ in the dual cognitive task (Table 4). Only $7.7 \%$ of the participants need 3 seconds and above to complete turning in the dual cognitive task.

Table 4. Percentage of time taken to complete turn under dual-task conditions for stroke survivors and healthy controls

\begin{tabular}{|c|c|c|c|c|c|c|c|c|c|c|}
\hline \multirow{2}{*}{$\begin{array}{l}\text { Time Taken during Turning } \\
\text { Category }\end{array}$} & \multicolumn{5}{|c|}{ Stroke } & \multicolumn{5}{|c|}{ Healthy Control } \\
\hline & $\begin{array}{l}\text { Single-Task } \\
(\%)\end{array}$ & $\begin{array}{l}\text { Dual } \\
\text { Task (\%) }\end{array}$ & Motor & $\begin{array}{l}\text { Dual } \\
\text { Task (\%) }\end{array}$ & Cognitive & $\begin{array}{l}\text { Single-Task } \\
(\%)\end{array}$ & $\begin{array}{l}\text { Dual } \\
\text { Task (\%) }\end{array}$ & Motor & $\begin{array}{l}\text { Dual } \\
\text { Task (\%) }\end{array}$ & Cognitive \\
\hline $\begin{array}{l}\text { 0-Complete turn in less than } 2.50 \\
\text { seconds }\end{array}$ & 0 & 0 & & 0 & & 0 & 0 & & 0 & \\
\hline $\begin{array}{l}\text { 1- Complete turn in } 2.50 \text { to } 2.99 \\
\text { seconds }\end{array}$ & 0 & 0 & & 0 & & 100 & 100 & & 91.7 & \\
\hline $\begin{array}{l}\text { 2- Accomplishes turn in } 3.00 \\
\text { seconds above }\end{array}$ & 100 & 100 & & 100 & & 0 & 0 & & 7.7 & \\
\hline
\end{tabular}

\subsection{Strategy used to complete turn}

Table 5 shows the strategy used to complete turning. Most of the stroke survivors are mainly found to use mixed strategy to complete $180^{\circ}$ turn with $61.5 \%$ in dual motor and dual cognitive task while $53.8 \%$ in a single task. Moreover, the strategy employed by the stroke survivors is steps with the score of $46.2 \%$ on a single task and $38.5 \%$ in both dual motor and dual cognitive task. In comparison, the healthy controls usually are observed to use pivot strategy to complete turn that includes $91.7 \%$ for both single and dual motor task whereas $83.3 \%$ for the dual cognitive task. The mixed strategy is the least strategy used to complete $180^{\circ}$ turn that is $16.7 \%$ for the dual cognitive task and $8.3 \%$ in both single and dual motor task.

Table 5. Percentage of strategy used to complete turn under dual-task conditions in stroke survivors and healthy controls

\begin{tabular}{|c|c|c|c|c|c|c|c|}
\hline \multicolumn{2}{|c|}{ Strategy Used to Complete Turn } & \multicolumn{2}{|l|}{ Stroke } & \multicolumn{4}{|c|}{ Healthy Control } \\
\hline \multirow{3}{*}{ Category } & \multirow{3}{*}{ Description } & Single- & Dual & Dual & Single- & Dual & Dual \\
\hline & & Task (\%) & Motor & Cognitive & Task (\%) & Motor & Cognitive \\
\hline & & & Task (\%) & Task (\%) & & Task $(\%)$ & Task (\%) \\
\hline \multirow[t]{2}{*}{ Pivot } & The body rotates/spins 180 degrees as a unit (en bloc) & & & & & & \\
\hline & $\begin{array}{l}\text { over the foot (or feet) in one controlled, discrete } \\
\text { movement; the foot (or feet) acts as a pivot point about } \\
\text { which the body spins } 180 \text { degrees. }\end{array}$ & 0 & 0 & 0 & 91.7 & 91.7 & 83.3 \\
\hline \multirow[t]{2}{*}{ Mixed } & Partial rotations accomplish 180 -degree turn or spinning & & & & & & \\
\hline & $\begin{array}{l}\text { mixed with steps or weight shifts were no pivoting occurs. } \\
\text { A mixture of steps and pivot movements occurs }\end{array}$ & 53.8 & 61.5 & 61.5 & 8.3 & 8.3 & 16.7 \\
\hline Steps & $\begin{array}{l}\text { 180-degree turn is accomplished with no evidence of } \\
\text { pivoting or spinning; the turn is accomplished through a } \\
\text { series of steps or weight shifts }\end{array}$ & 46.2 & 38.5 & 38.5 & 0 & 0 & 0 \\
\hline
\end{tabular}

\section{Discussion}

The aim of this study was to determine turning difficulty characteristics in chronic stroke survivors and healthy controls. We presented few important findings. First, both stroke survivors and healthy controls presented without staggering during $180^{\circ}$ turn. Second, stroke survivors presented with turning difficulty that they exhibited nearly more than five steps to accomplish $180^{\circ}$ turn for more than 3 seconds. In contrast, healthy controls took 1 to 2 steps and within 2.50 to 2.99 seconds to complete a turn. 
Lastly, the strategy used by the stroke survivors to complete the task was of mixed steps while the healthy gender-matched controls used pivot steps.

We found that stroke survivors did not exhibit staggering while performing 180 turns in all tasks. Although few stroke survivors were presented with slight staggering during the turn, however, they managed to self-correction without assistance. One possible explanation for this finding may be related to the duration of post-stroke. In this study, participants were more than 6 months post-stroke and usually the recovery of motor function in the paretic leg restore within 3 months (Geurts, de Haart, van Nes, \& Duysens, 2005). As a result, stroke survivors were able to complete turning without any falls incidence although when they were required to perform two tasks simultaneously. Despite that, all the healthy controls obtain $100 \%$ with no staggering in all task as they have normal balance reaction.

Our results demonstrated that stroke survivors used more than five steps during $180^{\circ}$ turning in all the three tasks. It also acts as the indicator of turning difficulty. The changes in movement strategy may appear in response due to loss of balance when performing the turn. Stroke survivors with impaired functional balance (mean BBS score: 45) may adopt the stepping strategy by taking a greater number of steps along with an increase in stride width for safety. When performing two tasks simultaneously, stroke survivors must prioritize attention to the secondary task, and they do not have gait as an automatic skill. Therefore, the deficits found on turning performance might be explained by the necessity to use cortical resources to perform the task. In addition, stroke survivors may require greater attentional resources during walking to compensate for their impaired sensorimotor function and balance. Previous study have reported that older adults with turning difficulty used multiple steps (5 or more), showed marked hesitancy, pauses, and stops while turning (Thigpen et al., 2000). This study also demonstrated that healthy controls using 1 to 2 steps while turning in a single task and dual motor task. However, they used more than 5 steps in the dual cognitive task. This result suggested that some of the healthy controls were presented with turning difficulty under dual cognitive task. Our data are best explained by the capacity sharing theory. The capacity-sharing theory assumes that attentional resources are fixed in capacity, and task performance depends on the amount of resources that are allocated to the task (YogevSeligmann, Hausdorff, \& Giladi, 2008). Under a dual-task condition, deterioration of task performance will be observed as attentional resources that are allocated to both tasks are reduced. The deterioration of performance will be observed in either one or both tasks, depending on task prioritization strategy. To compensate this problem, they tend to make smaller, simpler and slower movements that achieve the same goal that is to complete the turn.

In the present study, the time taken to complete $180^{\circ}$ turn was categorized into the following: (1) less than 2.5 seconds, (2) 2.5 to 2.99 seconds and (3) 3 seconds and above. We found that stroke survivors were found using 3 seconds and above to complete the turning in all tasks required. The findings are consistent with a previous study in which performing turning under dual-task conditions had led to increasing the time taken (Manaf, Justine, Goh, \& Latiff, 2014). This finding suggested that longer time to turn was an indicator of turning difficulty and risk of fall (Thigpen et al., 2000). During a $180^{\circ}$ turning, participants may slow down their gait speed and pause or stop between steps, and then they start walking again after that. These occur as a protective mechanism and as a precaution in preventing loss of balance that can lead to fall. In contrast, the healthy controls took 2.50 to 2.99 seconds to complete the turning in all three conditions.

The turning strategies were categorized into pivot, mixed and steps turn. Step turning involves a change in the direction to the opposite side of the stance limb, whereas a pivot turn implies a change in the direction towards the stance limb. Another interesting observation in this study was that the stroke survivors mainly used mixed strategy and steps to complete $180^{\circ}$ turn in single, dual motor and dual cognitive tasks. This mixed strategy was characterized by the absence of pivoting with multiple steps and weight shift toward one side. In contrast, the healthy controls were used pivot strategy to complete turning tasks in all three conditions. From these two groups, it can be inferred that stroke survivors lack in pivot strategies while turning. Multiple steps were used as a turning strategy to increase both stability and balance during the turn. Stroke survivors do not use pivot strategy during $180^{\circ}$ turn because they move in an "en bloc" pattern, which the body turns into one block in one direction (Hollands et al., 2010). The "en bloc" occurs as one block of body with their neck and trunk rigid, involving in multiple small steps to accomplish a turn rather than the usual twisting of the neck, trunk and pivoting on the toes (Verheyden, Willems, Ooms, \& Nieuwboer, 2007). Therefore, differences in the strategy indicate that the possible changes in gait occur to minimize instability during the turn.

This study has several limitations. First, the current findings obtained from a relatively small sample size and probably cannot be generalized to all stroke survivors. Second, we did not analyze the gait performance using a motion analysis system that would allow us to determine the kinematic changes in gait parameters. Future study may highlight the interplay between cognitive and biomechanical systems in controlling turning performance in chronic stroke. Lastly, the procedure was conducted in a physiotherapy gymnasium (controlled environment) that might not be representative of a regular environment encountered by the stroke survivors on a daily basis. 


\section{Conclusion}

Skilled turns during walking constitute a critical factor for safe ambulation. Turning difficulty is a common activity limitation in stroke survivors. These deteriorations of gait performances were prominent and exaggerated under dual tasks conditions. The results of this investigation show that stroke survivors had difficulty performing a turn and a secondary task simultaneously. Patients with stroke demonstrate turning deficits based on longer time and more steps to finish a turn. In contrast, the healthy gender-matched controls presented with no staggering, but took only 1 to 2 steps to complete the turn and needed 2.50-2.99 seconds to accomplish the turning task. The stroke survivors were using the mixed steps strategy while walking while the healthy gender-matched controls were using pivot steps as the strategy. The qualities for turning across the three conditions were the same pattern both in stroke and in healthy controls. Our results also provide a few important clinical implications for stroke assessment and rehabilitation. The ability to carry out dual tasks after stroke is essential to ensure a safe and efficient gait, especially in outdoor settings. Thus, it is recommended that dual-task turning may be incorporated into gait assessment that could serve as the foundation for the development of fall prevention strategy for stroke survivors. In addition, it could provide information towards the barrier-free facilities in addition to straight walking, ascending and descending stairs and walking over an uneven surface.

\section{Acknowledgements}

The authors wish to thank the Ministry of Education, Malaysia for funding the research project through the Research Acculturation Grant Scheme [Ref. No. 600-RMI/RAGS 5/3 (68/2014)] and, the Research Management Institute (RMI), Universiti Teknologi MARA (UiTM) for the administrative support.

\section{References}

Abbas, M. Y., \& Saruwono, M. (2012). Our 'Golden' Citizens with 'Golden' Facilities? Procedia - Social and Behavioral Sciences, 49(0), $127-146$.

Chen, H., Chen, C., Huang, W., Chung, Y., \& Tang, P. (2007). Development of a clinical measure of $180^{\circ}$ turning for post-stroke hemiplegic patients. Journal of Biomechanics, 40, S443.

Cumming, R. G., \& Klineberg, R. J. (1994). Fall frequency and characteristics and the risk of hip fractures. Journal of American Geriatrics Society, $42(7), 774-778$.

Eusuf, M. A., Mohit, M. A., Eusuf, M. M. R. S., \& Ibrahim, M. (2014). Impact of Outdoor Environment to the Quality of Life. Procedia - Social and Behavioral Sciences, 153(0), 639-654.

Geurts, A. C., de Haart, M., van Nes, I. J., \& Duysens, J. (2005). A review of standing balance recovery from stroke. Gait \& posture, 22(3), $267-281$.

Hollands, K. L., Hollands, M. A., Zietz, D., Wing, A. M., Wright, C., \& Van Vliet, P. (2010). Kinematics of turning 180 during the timed up and go in stroke survivors with and without falls history. Neurorehabilitation and Neural Repair, 24(4), 358-367.

Hyndman, D., Ashburn, A., \& Stack, E. (2002). Fall events among people with stroke living in the community: circumstances of falls and characteristics of fallers. Arch Phys Med Rehabil, 83(2), 165-170.

Manaf, H., Justine, M., Goh, H.-T., \& Latiff, L. (2014). Comparison of Gait Parameters Across Three Attentional Loading Conditions During Timed Up and Go Test in Stroke Survivors. Topics in Stroke Rehabilitation, 21(2), 128-136.

Mohammad, N. M. N., \& Abbas, M. Y. (2012). Elderly Environment in Malaysia: Impact of Multiple Built Environment Characteristics. Procedia - Social and Behavioral Sciences, 49(0), 120-126.

Mohit, M. A. (2014). Present Trends and Future Directions of Quality-of-Life. Procedia - Social and Behavioral Sciences, 153(0), 655-665.

Osman, M. M., Radzi, F. H. M., Bakri, N. I. M., \& Ibrahim, M. (2015). Barrier-free Campus: University Malaya, Kuala Lumpur. Procedia - Social and Behavioral Sciences, 168(0), 134-144.

Samah, Z. A., Ibrahim, N., \& Amir, J. S. (2013). Translating Quality Care Factors to Quality Space: Design Criteria for Outpatient Facility. Procedia - Social and Behavioral Sciences, 105(0), 265-272.

Shumway-Cook, A., Brauer, S., \& Woollacott, M. (2000). Predicting the probability for falls in community-dwelling older adults using the Timed Up \& Go Test. Physical Therapy, 80(9), 896-903.

Thigpen, M. T., Light, K. E., Creel, G. L., \& Flynn, S. M. (2000). Turning difficulty characteristics of adults aged 65 years or older. Physical Therapy, 80(12), 11741187.

Verheyden, G., Willems, A.-M., Ooms, L., \& Nieuwboer, A. (2007). Validity of the trunk impairment scale as a measure of trunk performance in people with Parkinson's disease. Archives of Physical Medicine and Rehabilitation, 88(10), 1304-1308.

Yates, J. S., Lai, S. M., Duncan, P. W., \& Studenski, S. (2002). Falls in community-dwelling stroke survivors: an accumulated impairments model. Journal of Rehabilitation Research \& Development, 39(3), 385-394.

Yogev-Seligmann, G., Hausdorff, J. M., \& Giladi, N. (2008). The role of executive function and attention in gait. Movement Disorders, 23(3), $329-342$.

Zakaria, J., \& Ujang, N. (2015). Comfort of Walking in the City Center of Kuala Lumpur. Procedia - Social and Behavioral Sciences, 170(0), 642-652 